\title{
THE SCIENCE OF VACCINE ADJUVANTS: ADVANCES IN TLR4 LIGAND ADJUVANTS
}

\author{
Steven G. Reed ${ }^{\mathrm{a}^{*}}$, Fan-Chi Hsu ${ }^{\mathrm{a}}$, Darrick A. Carter ${ }^{\mathrm{a}}$ and Mark T. Orr ${ }^{\mathrm{a}}$ \\ ${ }^{a}$ Infectious Disease Research Institute, 1616 Eastlake Ave E, Seattle, WA 98102, USA \\ *Steven Reed: sreed@idri.org
}

\begin{abstract}
TLR ligands are used in modern vaccine adjuvants, TLR4 ligand-based adjuvants are the most advanced in commercial vaccines. Increased understanding of TLR4 receptor-ligand interactions enables chemical synthesis and modification of new leads and our understanding of the biological/immunological mechanisms of combination adjuvants enables formulation of potent and safe vaccine compositions. Characterization of non-glycolipid TLR4 ligands provided new mechanistic information that could lead to new formulations. This review discusses advances in TLR4 agonist design - both glycolipid and nonglycolipid based TLR4 ligands - as well as CD14 activation as options to activate or synergize with TLR4 signaling. Finally, we review the molecular and cellular mechanisms that are elicited by formulated TLR4 targeted combination adjuvants during the initiation of innate immune responses leading to quality adaptive responses.
\end{abstract}

\section{Outline}

1. Introduction

2. Advances in TLR4 ligand design

3. Non-glycolipid based TLR4 ligands

4. CD14 activation

5. Mechanisms of adjuvant activity of formulated TLR4 adjuvants

\section{Introduction}

Inducing durable adaptive immunity is essential for effective vaccines. Without the addition of adjuvants, newer, defined vaccines devoid of microbial components are less likely to trigger the appropriate combination of innate immune signals leading to quality adaptive responses. From generating effective $T$ cell responses, increasing antibody titers, durability, functionality and breadth, modern, targeted adjuvant formulations can provide versatility and desired results. This review will discuss recent advances in adjuvant design that target immunological activity and mechanisms of adjuvant action.

\section{Advances in TLR4 Ligand Design}

Innate sensing of molecular patterns derived from microbes - pathogenic and non-pathogenic - results in the activation of immune cells. Early studies by Johnson, et al. demonstrated that lipopolysaccharide 
(LPS) derived from gram-negative bacteria had potent adjuvant properties [1], but also had undesirable side effects limiting the potential as an adjuvant or therapeutic. Thus began studies that formed the basis for the development of TLR4-based adjuvants involving chemistry, biology, and formulation engineering. Ribi and others developed Monophosphoryl Lipid A (MPL), a disaccharide glycolipid, the first TLR ligand to be deliberately included in approved vaccines [2]. Past vaccines made from inactivated and lysed organisms had a number of innate agonists and their inclusion was grandfathered in as TLR and non-TLR innate ligands, but the first that included these by purposeful addition, and received regulatory licensure, were the skin cancer vaccine Melacine ${ }^{\mathrm{TM}}$, approved in Canada for melanoma therapy in 1999, followed by GSK's Fendrix ${ }^{\circledR}$ and Cervarix ${ }^{\circledR}$ for Hepatitis B and HPV, respectively $[3,4]$. Later began the development and clinical use of synthetic disaccharide glycolipids, including glucopyranosyl lipid A (GLA) (also known as 3DPHAD) [5].

To be useful as adjuvants TLR4 ligands must be properly formulated. Formulation of GLA in alum or stable nanoemulsion (SE) increases the magnitude of antibody responses to vaccine antigens and maintain the diverse IgG1/IgG2 response driven by GLA [6]. The increased antibody response with GLA$S E$ reflects expanded numbers of germline BCRs recruited into the response, not just expansion of a few clones [7]. Compared to SE, GLA-SE augments both the frequency of HA-specific IgG2 antibodies and the overall diversity of HA proteins recognized by vaccinated serum (e.g. vaccination with H5+GLA-SE produces anti-H1 or $\mathrm{H} 7$ antibodies) which may increase the efficacy of flu vaccines against drifted or shifted strains [8]. GLA and alum synergize to induce T-bet-expressing, IFN- $\gamma$-producing CD4 T cell and this synergy is much more robust between GLA and SE [6,9]. In humans, GLA-SE promotes production of TH1 cells and IgG1 and IgG3 responses to a recombinant vaccine antigen [10]. In a phase 1 clinical trial with the pandemic influenza antigen H5, GLA-SE augmented functional antibody titers and promoted sero-conversion while providing dose-sparing effects [11].

\section{Table 1 highlights some of the adjuvant properties of GLA based adjuvants.}

\begin{tabular}{|l|l|}
\hline Actions & References \\
\hline Local Innate Immune Responses Leading to Rapid Immunity & {$[12-14]$} \\
\hline Safe Immune Enhancement & {$[10,15]$} \\
\hline $\begin{array}{l}\text { Efficient Antigen Presentation Through Dendritic Cell Activation, Enhanced } \\
\text { Phagocytosis and Endosomal Activity }\end{array}$ & {$[6,14,16,17]$} \\
\hline Intracellular Pathogen Killing & {$[10,18,19]$} \\
\hline $\begin{array}{l}\text { High Quality Humoral Immunity (Magnitude, Breadth, and Affinity of Antibody } \\
\text { Responses Enhanced) }\end{array}$ & {$[7,9,20]$} \\
\hline$T_{H}$ Biased Immune Response & {$[9,14,19,21]$} \\
\hline Very Low Doses are Required Making Deployment Safe and Cost Effective & {$[10,15]$} \\
\hline
\end{tabular}

While GLA was based on known natural structures and gained potency by being included in designer formulations it was attractive to also make a designer TLR4 ligand. The crystal structure of the human 
TLR4 complex to MD-2 had been reported, facilitating inspection of molecular interactions [22]. Relatively minor modifications in the molecular structure led to significant changes in patterns of innate immune stimulation and the resulting molecule, SLA, a hexacyl structure with certain shortened acyl chain lengths, has shown properties distinct from those of GLA and has advanced through early stage clinical studies (Carter et al, submitted for publication) (Figure 1). Combining this with the inclusion of purely synthetic components into some leading adjuvant formulations is leading to a new generation of adjuvants that are designed from the ground up including the active agonist composition, synthetic excipients, and nanotechnological presentation of the formulation. Formulation of SLA in nanoemulsion or alum augmented TH1, functional antibodies, plasma cells and memory B cell responses against a recombinant protein and promoted protection against lethal viral challenge [23].

Recently, Amigorena and colleagues reported that cross-presentation in DCs is strongly influenced by LPS mediated TLR4 engagement. Mature DCs have less phagosome mediated degradation due to lower levels of lysosomal enzymes being recruited. Also, LPS-treated DCs have peri-nuclear clustering of lysosomes that leads to a strong reduction of phago-lysosomal fusion. The GTPase Rab34 controls lysosome organization; down-regulating Rab34 in LPS-treated DCs reverted the delayed phagosome maturation and thus increased cross-presentation [24].

\section{Non-glycolipid based TLR4 ligands}

Over the past few years several studies have demonstrated that microbial proteins can induce potent innate immune responses in antigen presenting cells, although the precise mechanism(s) responsible for this activity remain elusive. We previously identified that LeIF, a Leishmaniasis braziliensis gene homologue of the eukaryotic initiator factor $4 \mathrm{~A}$, can induce strong IL-12 and TNF- $\alpha$ production in macrophages and DCs and thereby favors developing a TH1 biased immune response [25,26]. More recently, we have found that LelF may signal through both TLR2 and TLR4 (Reed et al., in preparation). Furthermore, in the presence of CD14-blocking antibodies, LelF-induced IL- 6 and MIP-1 $\beta$ production by Mono Mac 6 cells was diminished whereas similar treatment of GLA-stimulated cells did not inhibit cytokine production. MD-2 is not required for LelF-induced TLR4 activation as MD-2 blocking antibody has no effect on cytokine production of Mono Mac 6 in the presence of LeIF. Our data suggest that LeIF acts through CD14 to signal to both TLR2 complexes and to TLR4, and its action is distinct from LPSderived agonists. The exact mechanism of how LelF binds to CD14 is currently under investigation.

Beutler and colleagues recently reported that a synthetic peptidomimetic compound, Neoseptin-1, and its chemical modified relatives, Neoseptin-3 and Neoseptin-4, could bind and activate the mouse TLR4/MD-2 complex independent of LPS [27]. Neoseptin-3 induced strong TNF, IL-6 and IFN- $\gamma$ productions in myeloid cells as well as activated NF-KB, MAPK, and TBK1 to levels comparable to those obtained through LPS-mediated TLR4 activation. Neoseptin-3 forms a dimer and directly binds to the hydrophobic pocket of MD-2. Although the residue contacts of MD-2 to Neoseptin-3 are distinct from those that mediate binding of LPS or lipid A, Neoseptin-3 triggers a conformational change similar to that induced by natural ligands of TLR4. These recent findings suggest that non-glycolipid molecules could be developed into TLR4 agonist adjuvants. A comparison of binding preference among MPL, 
Neoseptin-3 and LeIF is shown in Figure 2. Thus, there is a growing body of evidence that non glycolipid structures can activate immune responses via TLR4.

\section{CD14 activation}

Direct activation of CD14 may lead to a new generation of adjuvants. CD14 is a well-known cofactor for TLR4 and TLR2/TLR6, but it can also activate TLR3, TLR7 and TLR9. The N-terminus of human and mouse CD14 is important for transferring LPS to signal to TLR4 $[28,29]$. CD14 uses a similar binding pocket to interact with various microbial products including $\mathrm{Pam}_{3} \mathrm{CSK}_{4}$, peptidoglycan, lipoteichoic acid (LTA), polyl: $C$ and CpG DNA [30]. Thus, by interacting with CD14, an adjuvant/agonist could synergistically stimulate multiple TLR leading to potent adaptive immunity. Studies are starting to elucidate how CD14 mediated signaling to TLRs, mainly TLR4, is regulated. Upon LPS stimulation, CD14 is crucial for inducing inflammatory endocytosis that leads TLR4 to endosomes and triggers downstream MyD88 and TRIF pathways [31]. The endocytosis of TLR4 is promoted by the extracellular protein MD-2, but not by TLR4's cytosolic tail. Most importantly, monophosphorylated LPS derived from commensal or pathogenic bacteria triggers less CD14 activation, TLR4/MD-2 dimerization and endocytosis as compared to diphophorylated LPS [31]. The synergy between CD14 and thrombomodulin [32] or complement C5 [33] that leads to TLR4 activation and inflammatory responses also suggests that switching CD14 activation ON/OFF may be crucial to designing next generation adjuvants.

\section{Mechanisms of adjuvant activity of formulated TLR4 adjuvants}

Alum, emulsion, and saponin (e.g. QuilA or QS21) adjuvants all require components of the inflammasome cascade to augment humoral immunity [34-36]. Activation of inflammasomes occur by diverse stimuli including pathogen- and host-derived molecules recognized by pattern recognition receptors including the NLRP family and AIM2. Known triggers include cytoplasmic dsDNA, extracellular ATP, uric acid and other DAMPs. [37]. Upon binding these DAMPs, the NLRP family and AIM2 receptors recruit the adapter protein ASC, which causes the oligomerization and activation of caspase-1 and/or caspase-11. Caspase-1/11 converts pro-IL-1 $\beta$, IL-18, and IL-33 into their active forms, which are subsequently secreted. These cytokines shape the innate and adaptive responses to pathogens and immunization [38].

TLR signaling increases the expression of inflammasome components and production of the procytokine substrates of caspase 1 including pro-IL-1 and pro-IL-18, thus, TLR and DAMP signaling may converge to enhance production of the mature forms of these key cytokines [38]. For the oil-in-water TLR4 agonist formulation, GLA-SE, caspase-1/11 is necessary to produce mature IL-18. IL-18 then drives the production of IFN- $\gamma$ by innate cells including NK cells and neutrophils, as well as memory CD8 T cells, that in turn promotes TH1 immunity [12]. Similarly, the combination adjuvant AS01 (MPL formulated with the saponin QS21 in liposomes) rapidly induces IL-1 $\beta$ production in vivo at both the injection site and in the draining lymph node [39]. Other adjuvant candidates with different mechanisms such as cholera toxin and the $E$. coli-derived, attenuated heat labile toxin, $\mathrm{dmLT}$, activate the inflammasome to augment cellular immune responses when used as an adjuvant [40]. This suggests that the inflammasome pathway may be universally important for the activity of successful vaccine adjuvants. 
Additionally both GLA-SE and ASO1 produce a massive influx of neutrophils into the injection site and draining lymph node and depend on antigen presentation by dendritic cells to promote cellular immune responses $[12,39,41]$. Although GLA-SE and AS01 incorporate different TLR4 agonists and different DAMP-adjuvants, they appear to rely on similar mechanisms of action to promote robust cellular and/or humoral immune responses.

Recent work has pointed to an additional innate immune cell type, the subcapsular macrophage, playing a key role in the action of several adjuvants. MF59 causes retention of unprocessed antigen in the draining lymph node where it is captured by subcapsular macrophages. This in turn promotes deposition of immune complexes of unprocessed antigen onto follicular dendritic cells, which are important for promoting humoral immunity [42]. Disruption of the subcapsular macrophage network impaired the adaptive immune responses promoted by TLR agonists, implicating this network in the adjuvanticity of TLR agonist containing adjuvants [43]. Depletion of these cells was sufficient to impair the cellular and humoral responses to GLA-SE adjuvanted immunization (manuscript in preparation).

An increasing body of clinical data strongly supports including TLRL in subunit vaccines, with TLR4L being by far the most advanced. However, with new molecular mechanisms of TLR4 activation being defined and new classes of molecules being discovered, it is evident that there is still much to learn about this class of adjuvant compounds. 


\section{FIGURES}

\section{Figure 1. SLA Design and Putative Fit into TLR4/MD-2 pocket}

(A) Structure of SLA, red transparent area depicts acyl chain region targeted for better fit into the MD-2 pocket. (B) Crystal structure of human TLR4/human MD-2 (gray translucent surface) bound to a TLR4 agonist (golden van der Waals radii). The red arrow indicates the tight packing of acyl chains into the human MD-2 pocket. This crowding indicated fewer carbons on these acyl chains would make for a better fit into the pocket and this region was modified to generate SLA. The green arrow indicates the exposed chain that explains the need for hexa-acylated ligands or at the very minimum this acyl position to be occupied for human TLR4 signaling. The exposed chain the arrow indicates is responsible for

completing the interface between two TLR4/MD-2 complexes and provides for an active signaling molecule. Antagonists of human TLR4 have either a short or no acyl chain in this position.

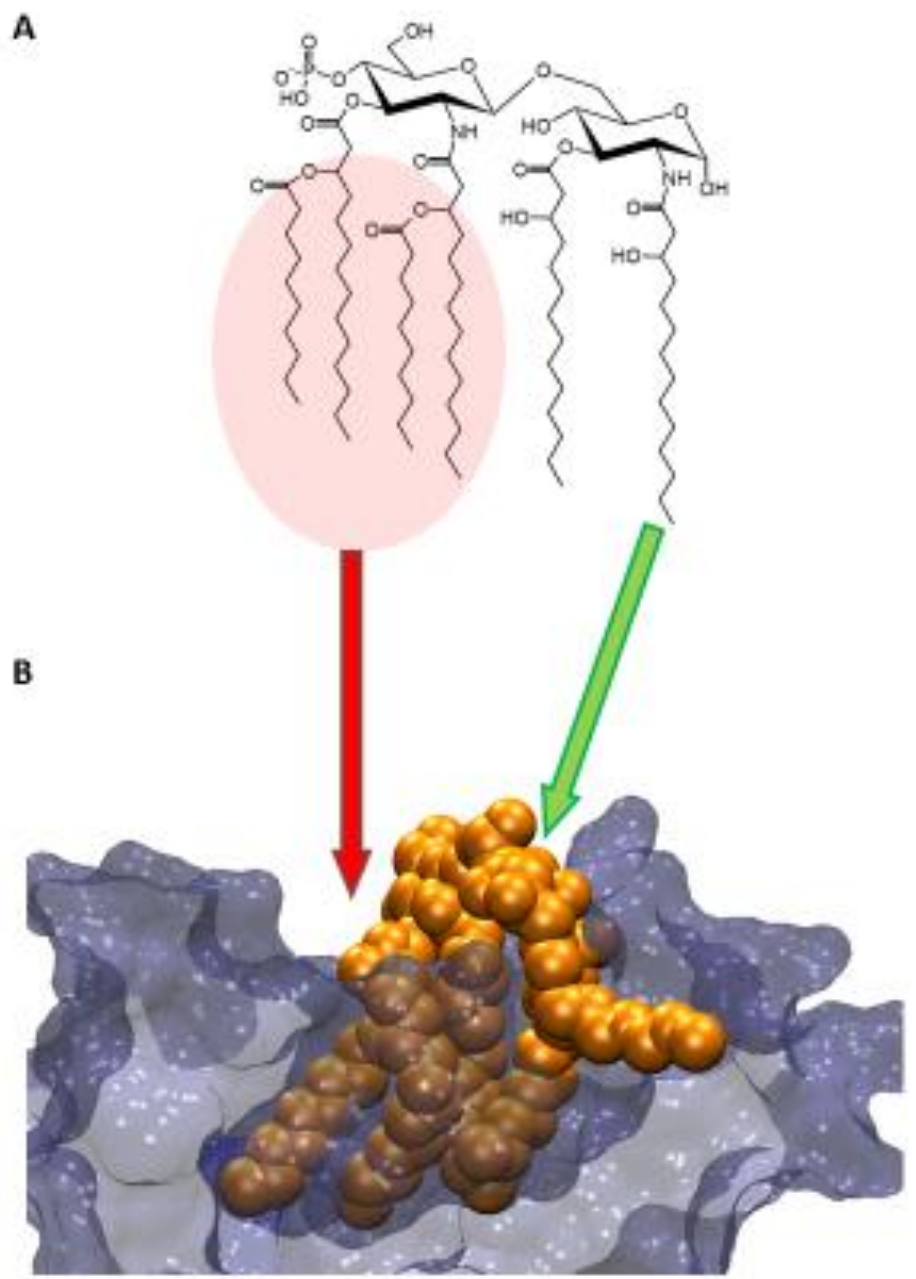


Figure 2. TLR4 agonists activate TLR4 signaling via different mechanisms.

(A) MD-2 and CD14 are required for TLR4 to bind lipopolysaccharide (LPS)-derived TLR4 agonist (e.g. MPL). Two CD14s form a horseshoe-shaped structure that anchors to the ectodomains of TLRs. Lipid chains of MPL are buried inside the hydrophobic pocket of MD-2 that results in the conformational change of MD-2 and triggers TLR4 signaling. (B) Synthetic non-glycolipid TLR4 agonist Neoseptin-3 activates mouse TLR4-MD-2 complex, but it does not require CD14. Neoseptin-3 directly occupies hydrophobic pocket of MD-2 and its phenol ring interacts residues of MD-2 and TLR4 that are distinct to those for LPS. (C) LelF is a Leishmania braziliensis protein that is homologous to eukaryotic initiator factor 4A, a RNA helicase. The N-terminal portion of LelF can act as a natural non-glycolipid TLR4 agonist by binding to CD14. MD-2 is not required for LelF-induced TLR4 activation. In addition, by interacting with CD14, LeIF may activate TLR2-TLR6 and TLR2-TLR1 complexes.

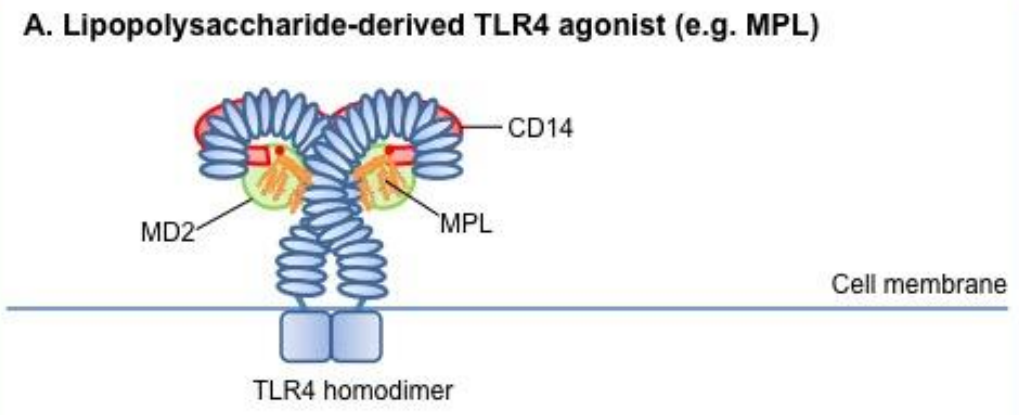

B. Synthetic non-glycolipid TLR4 agonist (e.g. Neoseptin-3)

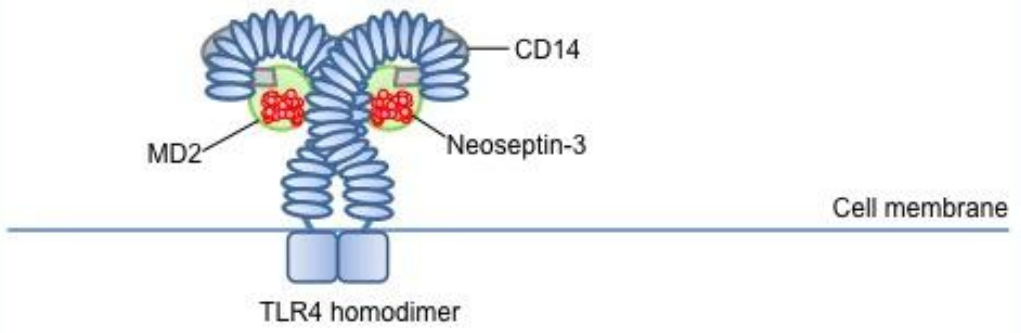

C. Natural non-glycolipid TLR4 agonist (e.g. LelF)

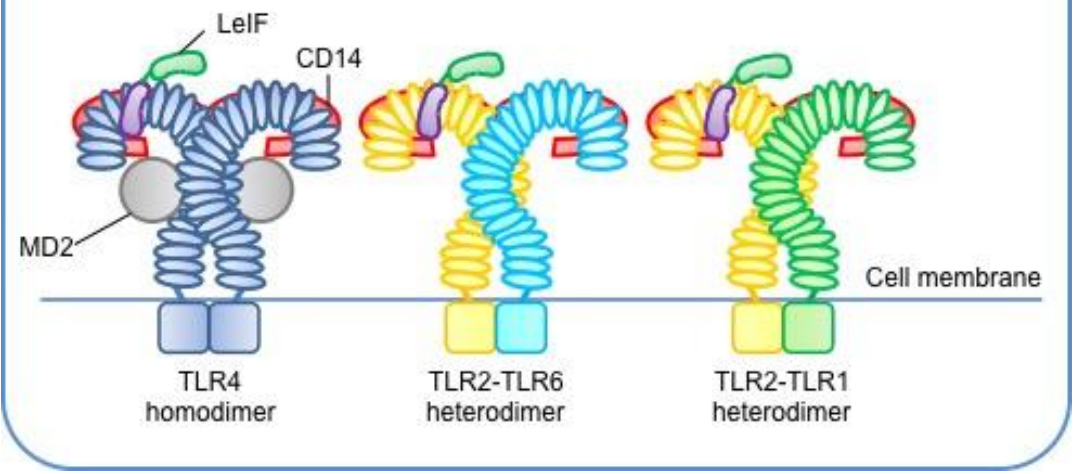




\section{ACKNOWLEDGEMENTS}

This research was supported with funding from the Bill \& Melinda Gates Foundation, under grant number OPP1130379 and by grant R01-AI-025038 from the National Institute of Allergy And Infectious Diseases of the National Institutes of Health to SGR. The content is solely the responsibility of the authors and does not necessarily represent the official views of the National Institutes of Health.

\section{REFERENCES}

1. Johnson AG, Gaines S, Landy M: Studies on the $\mathbf{O}$ antigen of Salmonella typhosa. V. Enhancement of antibody response to protein antigens by the purified lipopolysaccharide. J Exp Med 1956, 103:225-246.

2. Johnson DA: TLR4 agonists as vaccine adjuvants: a chemist's perspective. Expert Rev Vaccines 2013, 12:711-713.

3. Beran J: Safety and immunogenicity of a new hepatitis B vaccine for the protection of patients with renal insufficiency including pre-haemodialysis and haemodialysis patients. Expert Opin Biol Ther 2008, 8:235-247.

4. Schwarz TF: Clinical update of the AS04-adjuvanted human papillomavirus-16/18 cervical cancer vaccine, Cervarix. Adv Ther 2009, 26:983-998.

5. Coler RN, Bertholet S, Moutaftsi M, Guderian JA, Windish HP, Baldwin SL, Laughlin EM, Duthie MS, Fox CB, Carter D, et al.: Development and characterization of synthetic glucopyranosyl lipid adjuvant system as a vaccine adjuvant. PloS one 2011, 6:e16333.

6. Orr MT, Fox CB, Baldwin SL, Sivananthan SJ, Lucas E, Lin S, Phan T, Moon JJ, Vedvick TS, Reed SG, et al.: Adjuvant formulation structure and composition are critical for the development of an effective vaccine against tuberculosis. Journal of controlled release : official journal of the Controlled Release Society 2013, 172:190-200.

7. Wiley SR, Raman VS, Desbien A, Bailor HR, Bhardwaj R, Shakri AR, Reed SG, Chitnis CE, Carter D: Targeting TLRs expands the antibody repertoire in response to a malaria vaccine. Sci Transl Med 2011, 3:93ra69.

8. Desbien AL, Van Hoeven N, Reed SJ, Casey AC, Laurance JD, Baldwin SL, Duthie MS, Reed SG, Carter D: Development of a high density hemagglutinin protein microarray to determine the breadth of influenza antibody responses. Biotechniques 2013, 54:345-348.

9. Baldwin SL, Fox CB, Pallansch MA, Coler RN, Reed SG, Friede M: Increased potency of an inactivated trivalent polio vaccine with oil-in-water emulsions. Vaccine 2011, 29:644-649.

10. Coler RN, Duthie MS, Hofmeyer KA, Guderian J, Jayashankar L, Vergara J, Rolf T, Misquith A, Laurance JD, Raman VS, et al.: From mouse to man: safety, immunogenicity and efficacy of a candidate leishmaniasis vaccine LEISH-F3+GLA-SE. Clin Transl Immunology 2015, 4:e35.

11. Treanor JJ, Essink B, Hull S, Reed S, Izikson R, Patriarca P, Goldenthal KL, Kohberger R, Dunkle LM: Evaluation of safety and immunogenicity of recombinant influenza hemagglutinin (H5/Indonesia/05/2005) formulated with and without a stable oil-in-water emulsion containing glucopyranosyl-lipid A (SE+GLA) adjuvant. Vaccine 2013, 31:5760-5765.

12. Desbien AL, Reed SJ, Bailor HR, Dubois Cauwelaert N, Laurance JD, Orr MT, Fox CB, Carter D, Reed SG, Duthie MS: Squalene emulsion potentiates the adjuvant activity of the TLR4 agonist, GLA, via inflammatory caspases, IL-18, and IFN-gamma. Eur J Immunol 2015, 45:407-417.

** This paper described the interaction between emulsions and TLR agonists in enhancing the immune response. It shows that inflammasome activation by the emulsion components and TLR 
activation by the TLR4 agonist synergize through IFN-gamma, IL-18 and the action of caspases providing a framework for designing new combination adjuvants.

13. Fox CB, Mulligan SK, Sung J, Dowling QM, Fung HW, Vedvick TS, Coler RN: Cryogenic transmission electron microscopy of recombinant tuberculosis vaccine antigen with anionic liposomes reveals formation of flattened liposomes. Int J Nanomedicine 2014, 9:1367-1377.

14. Lambert SL, Yang CF, Liu Z, Sweetwood R, Zhao J, Cheng L, Jin H, Woo J: Molecular and cellular response profiles induced by the TLR4 agonist-based adjuvant Glucopyranosyl Lipid A. PloS one 2012, 7:e51618.

15. Santini-Oliveira M, Coler RN, Parra J, Veloso V, Jayashankar L, Pinto PM, Ciol MA, Bergquist R, Reed SG, Tendler M: Schistosomiasis vaccine candidate Sm14/GLA-SE: Phase 1 safety and immunogenicity clinical trial in healthy, male adults. Vaccine 2016, 34:586-594.

16. Schneider LP, Schoonderwoerd AJ, Moutaftsi M, Howard RF, Reed SG, de Jong EC, Teunissen MB: Intradermally administered TLR4 agonist GLA-SE enhances the capacity of human skin DCs to activate $T$ cells and promotes emigration of Langerhans cells. Vaccine 2012, 30:4216-4224.

17. Misquith A, Fung HW, Dowling QM, Guderian JA, Vedvick TS, Fox CB: In vitro evaluation of TLR4 agonist activity: formulation effects. Colloids Surf B Biointerfaces 2014, 113:312-319.

18. Windish HP, Duthie MS, Misquith A, Ireton G, Lucas E, Laurance JD, Bailor RH, Coler RN, Reed SG: Protection of mice from Mycobacterium tuberculosis by ID87/GLA-SE, a novel tuberculosis subunit vaccine candidate. Vaccine 2011, 29:7842-7848.

19. Cha SB, Kim WS, Kim JS, Kim H, Kwon KW, Han SJ, Cho SN, Coler RN, Reed SG, Shin SJ: Pulmonary immunity and durable protection induced by the ID93/GLA-SE vaccine candidate against the hyper-virulent Korean Beijing Mycobacterium tuberculosis strain K. Vaccine 2016, 34:21792187.

20. Lambert SL, Aslam S, Stillman E, MacPhail M, Nelson C, Ro B, Sweetwood R, Lei YM, Woo JC, Tang RS: A novel respiratory syncytial virus (RSV) $F$ subunit vaccine adjuvanted with GLA-SE elicits robust protective TH1-type humoral and cellular immunity in rodent models. PLoS One 2015, 10:e0119509.

21. Orr MT, Duthie MS, Windish HP, Lucas EA, Guderian JA, Hudson TE, Shaverdian N, O'Donnell J, Desbien AL, Reed SG, et al.: MyD88 and TRIF synergistic interaction is required for TH1-cell polarization with a synthetic TLR4 agonist adjuvant. Eur J Immunol 2013, 43:2398-2408.

22. Park BS, Allen DN, Barney SJ, Ringdahl EN, Mayfield J: Structure of attention in children with traumatic brain injury. Appl Neuropsychol 2009, 16:1-10.

23. Van Hoeven N, Joshi SW, Nana GI, Bosco-Lauth A, Fox C, Bowen RA, Clements DE, Martyak T, Parks DE, Baldwin S, et al.: A Novel Synthetic TLR-4 Agonist Adjuvant Increases the Protective Response to a Clinical-Stage West Nile Virus Vaccine Antigen in Multiple Formulations. PLoS One 2016, 11:e0149610.

* In this report the authors describe the ability of the next generation TLR4 agonist to enhance immune responses to viral pathogens.

24. Alloatti A, Kotsias F, Pauwels AM, Carpier JM, Jouve M, Timmerman E, Pace L, Vargas P, Maurin M, Gehrmann U, et al.: Toll-like Receptor 4 Engagement on Dendritic Cells Restrains PhagoLysosome Fusion and Promotes Cross-Presentation of Antigens. Immunity 2015, 43:1087-1100.

25. Skeiky YA, Guderian JA, Benson DR, Bacelar O, Carvalho EM, Kubin M, Badaro R, Trinchieri G, Reed SG: A recombinant Leishmania antigen that stimulates human peripheral blood mononuclear cells to express a Th1-type cytokine profile and to produce interleukin 12. J Exp Med 1995, 181:1527-1537. 
26. Probst P, Skeiky YA, Steeves M, Gervassi A, Grabstein KH, Reed SG: A Leishmania protein that modulates interleukin (IL)-12, IL-10 and tumor necrosis factor-alpha production and expression of B7-1 in human monocyte-derived antigen-presenting cells. Eur J Immunol 1997, 27:2634-2642.

27. Wang Y, Su L, Morin MD, Jones BT, Whitby LR, Surakattula MM, Huang H, Shi H, Choi JH, Wang KW, et al.: TLR4/MD-2 activation by a synthetic agonist with no similarity to LPS. Proc Natl Acad Sci U S A 2016, 113:E884-893.

**This study reported that a series of chemially synthesized peptides that bidn and activate the mouse TLR4/MD-2 complex indepdent of LPS. This is the first report of non-glycolid TLR4 agonist that triggers a similar conformational change as compared to LPS or lipid A, but uses distinct binding residues.

28. Kelley SL, Lukk T, Nair SK, Tapping RI: The crystal structure of human soluble CD14 reveals a bent solenoid with a hydrophobic amino-terminal pocket. J Immunol 2013, 190:1304-1311.

29. Ranoa DR, Kelley SL, Tapping RI: Human lipopolysaccharide-binding protein (LBP) and CD14 independently deliver triacylated lipoproteins to Toll-like receptor 1 (TLR1) and TLR2 and enhance formation of the ternary signaling complex. J Biol Chem 2013, 288:9729-9741.

30. Jin MS, Lee JO: Structures of the toll-like receptor family and its ligand complexes. Immunity 2008, 29:182-191.

31. Tan Y, Zanoni I, Cullen TW, Goodman AL, Kagan JC: Mechanisms of Toll-like Receptor 4 Endocytosis Reveal a Common Immune-Evasion Strategy Used by Pathogenic and Commensal Bacteria. Immunity 2015, 43:909-922.

**This study reported that CD14 is critical to promote TLR4 endocytosis, and MD-2 is a cargo-selection agent. It provides a novel mechanism that shows how pathogenic and commensal bacteria use monophosphorylated lipid A to escape inflammotry endocytosis pathway.

32. Ma CY, Chang WE, Shi GY, Chang BY, Cheng SE, Shih YT, Wu HL: Recombinant thrombomodulin inhibits lipopolysaccharide-induced inflammatory response by blocking the functions of CD14. $J$ Immunol 2015, 194:1905-1915.

33. Nymo S, Gustavsen A, Nilsson PH, Lau C, Espevik T, Mollnes TE: Human Endothelial Cell Activation by Escherichia coli and Staphylococcus aureus Is Mediated by TNF and IL-1beta Secondarily to Activation of C5 and CD14 in Whole Blood. J Immunol 2016, 196:2293-2299.

34. Wilson NS, Duewell P, Yang B, Li Y, Marsters S, Koernig S, Latz E, Maraskovsky E, Morelli AB, Schnurr $M$, et al.: Inflammasome-dependent and -independent IL-18 production mediates immunity to the ISCOMATRIX adjuvant. J Immunol 2014, 192:3259-3268.

35. Vono M, Taccone M, Caccin P, Gallotta M, Donvito G, Falzoni S, Palmieri E, Pallaoro M, Rappuoli R, Di Virgilio F, et al.: The adjuvant MF59 induces ATP release from muscle that potentiates response to vaccination. Proc Natl Acad Sci U S A 2013, 110:21095-21100.

36. Eisenbarth SC, Colegio OR, O'Connor W, Sutterwala FS, Flavell RA: Crucial role for the Nalp3 inflammasome in the immunostimulatory properties of aluminium adjuvants. Nature 2008, 453:1122-1126.

37. Jounai N, Kobiyama K, Takeshita F, Ishii KJ: Recognition of damage-associated molecular patterns related to nucleic acids during inflammation and vaccination. Front Cell Infect Microbiol 2012, 2:168.

38. Davis BK, Wen H, Ting JP: The inflammasome NLRs in immunity, inflammation, and associated diseases. Annu Rev Immunol 2011, 29:707-735. 
39. Didierlaurent AM, Collignon C, Bourguignon P, Wouters S, Fierens K, Fochesato M, Dendouga N, Langlet C, Malissen B, Lambrecht BN, et al.: Enhancement of adaptive immunity by the human vaccine adjuvant AS01 depends on activated dendritic cells. J Immunol 2014, 193:1920-1930.

40. Larena $M$, Holmgren J, Lebens $M$, Terrinoni $M$, Lundgren A: Cholera toxin, and the related nontoxic adjuvants $\mathrm{mmCT}$ and dmLT, promote human Th17 responses via cyclic AMP-protein kinase $A$ and inflammasome-dependent IL-1 signaling. J Immunol 2015, 194:3829-3839.

41. Pantel A, Cheong C, Dandamudi D, Shrestha E, Mehandru S, Brane L, Ruane D, Teixeira A, Bozzacco L, Steinman RM, et al.: A new synthetic TLR4 agonist, GLA, allows dendritic cells targeted with antigen to elicit Th1 T-cell immunity in vivo. Eur J Immunol 2012, 42:101-109.

42. Cantisani R, Pezzicoli A, Cioncada R, Malzone C, De Gregorio E, D'Oro U, Piccioli D: Vaccine adjuvant MF59 promotes retention of unprocessed antigen in lymph node macrophage compartments and follicular dendritic cells. J Immunol 2015, 194:1717-1725.

43. Gaya M, Castello A, Montaner B, Rogers N, Reis e Sousa C, Bruckbauer A, Batista FD: Host response. Inflammation-induced disruption of SCS macrophages impairs B cell responses to secondary infection. Science 2015, 347:667-672.

** This report describes the migration of certain macrophage populations in response to inflammatory signals and the resulting "shut-down" of the affected lymph node providing an interesting insight into why short term boosts may not enhance immunity and another mechanism by which inflammatory / adjuvant components are important to generating an immune response. 